\title{
PROFUNDIDADE E ATRIBUTOS FÍSICOS DO SOLO E SEUS IMPACTOS NAS RAÍZES DE BANANEIRAS ${ }^{1}$
}

\author{
ALAN ANTÔNIO MIOTTI², MIRIAN CRISTINA GOMES COSTA², \\ TIAGO OSÓRIO FERREIRA4 ${ }^{4}$, RICARDO ESPÍNDOLA ROMERO ${ }^{4}$
}

RESUMO - A bananeira pode ter sua produção prejudicada se cultivada em solos com restrições físicas. O objetivo deste trabalho foi verificar se os atributos físicos de solos rasos comprometem as raízes das plantas de bananeira. O estudo foi realizado na Chapada do Apodi (CE), onde foram comparados solos com duas profundidades efetivas: raso (profundidade aproximada de $0,57 \mathrm{~m}$, mais arenoso) e profundo (profundidade aproximada de $1,16 \mathrm{~m}$, mais argiloso) e seis profundidades de coleta de solo e de raízes $(0-10 ; 10-20 ; 20-30$; 30-40; 40-50 e 50-60 cm). Nas amostras de solo, foram determinados os seguintes atributos físicos: curva de retenção de água, densidade do solo, porosidade, granulometria e densidade de partículas. A resistência do solo à penetração (RP) foi avaliada no campo, utilizando-se de penetrômetro de impacto, com cinco repetições até $60 \mathrm{~cm}$ de profundidade. Para o estudo de raízes, as amostras foram coletadas com sonda e, por meio da análise de imagens de raízes lavadas, foram determinados: comprimento, área e volume total, e classes de diâmetro. O solo mais profundo e argiloso apresentou maior microporosidade e porosidade total, o que contribuiu para maior retenção de água. Valores de densidade do solo e de partículas foram maiores em duas camadas do solo raso, bem como a RP que atingiu o valor de 5,1 MPa. O desenvolvimento das raízes das bananeiras foi favorecido no solo profundo, no qual foram encontradas raízes mais espessas $(2,2$ a $6,6 \mathrm{~mm})$ e mais finas $(0,68$ a $0,79 \mathrm{~mm})$, contribuindo com a sustentação das plantas e com a absorção de água e nutrientes.

Termos para Indexação: Arquitetura radicular, Musa spp., bananicultura irrigada, Cambissolos.

\section{THE IMPACTS OF SOIL DEPTH AND PHYSICAL ATTRIBUTES ON BANANA PLANT ROOTS}

\begin{abstract}
The banana tree may have hampered its production if grown in soils with physical restrictions. The aim of this study was to verify if the physical attributes of shallow soils undermine the roots of banana tree plants. The study was conducted in the Apodi Plateau (CE), where soils were compared with two actual depths: shallow (approximately $0.57 \mathrm{~m}$ depth, more sandy) and deep (approximately $1.16 \mathrm{~m}$ depth, more clayey) and six sampling depths of soil and roots (0-10, 10-20, 20-30, 30-40, 40-50 and 50-60 cm). In the soil samples were determined the following physical attributes: water retention curve, bulk density, porosity, particle size and particle density. The soil resistance to penetration (RP) was assessed in the field using an impact penetrometer with five repetitions at $60 \mathrm{~cm}$ depth. To study the roots, samples were collected with a probe, and the washed roots were analyzed through images, being determined: length, area and total volume and diameter classes. The deep soil had higher clay content, higher microporosity and total porosity, which contributed to the higher water retention. Density of soils and particles were higher in two layers of the shallow soil, as well as the RP which reached 5.1 MPa. The roots of banana tree were favored in deep soil in which were found thicker ( 2.2 to $6.6 \mathrm{~mm})$ and thinner $(0.68$ to $0.79 \mathrm{~mm})$ roots, contributing to the support of plants and the absorption of water and nutrients.
\end{abstract}

Index terms: Root architecture; Musa spp., irrigated banana plantation, Cambisol.

\footnotetext{
${ }^{1}$ (Trabalho 025-12). Recebido em: 03-01-2012. Aceito para publicação em: 15-03-2013. Parte da dissertação de mestrado do primeiro autor.

${ }^{2}$ Engenheiro Agrônomo, Professor substituto, Faculdade de Ciências Biomédicas de Cacoal, Av. Cuiabá, 3087, CEP 76963-665, Cacoal-RO. Email: alan_miotti@hotmail.com

${ }^{3}$ Professor Adjunto, Departamento de Ciências do Solo, Universidade Federal do Ceará, Av. Mister Hull, 2977, CEP 60021-970, Fortaleza-CE. E-mail: mirian.costa@ufc.br (autor para correspondência).

${ }^{4}$ Professor Adjunto, Departamento de Ciências do Solo, Universidade Federal do Ceará, Av. Mister Hull, 2977, CEP 60021-970, Fortaleza - CE. E-mail: reromero@ufc.br
} 


\section{INTRODUÇÃO}

O Estado do Ceará é um importante produtor de bananas na região Nordeste do Brasil e parte dessa produção concentra-se no perímetro irrigado Jaguaribe-Apodi, localizado na Chapada do Apodi. A região destaca-se em meio ao semiárido por apresentar alto potencial agrícola devido à presença de solos originados de calcário, com boa fertilidade natural e relevo plano, favorável à mecanização (DNOCS, 2009). Os Cambissolos, classe dominante de solos na área, destacam-se por sua elevada variabilidade no que se refere às propriedades que afetam a produção das plantas (LEMOS et al., 1997).

Oliveira (2009), em estudo de caso no perímetro Jaguaribe-Apodi, verificou que a dinâmica da água, associada às variações no micrrorelevo, favoreceu a presença de Cambissolos rasos em superfícies convexas do terreno e profundos nas superfícies côncavas. Nos pontos mapeados como solo raso, a profundidade encontrada pelo autor variou de 0 a 30 $\mathrm{cm}$, enquanto nos pontos considerados mais profundos, a profundidade máxima foi de aproximadamente $100 \mathrm{~cm}$. Costa et al. (2011) avaliaram bananeiras que se desenvolveram nos pontos com solo raso e profundo, encontrando cachos com maior massa associados ao solo com maior profundidade efetiva.

Dentre os fatores que podem influenciar no desenvolvimento das raízes e, consequentemente, na absorção de água e nutrientes e no potencial produtivo da bananeira, destaca-se a profundidade efetiva do solo. De modo geral, valores inferiores a $25 \mathrm{~cm}$ de profundidade efetiva são considerados inadequados para alguns tipos de cultivo (BORGES; SOUZA, 2004). Para o bom desenvolvimento da bananeira, os autores recomendam que os solos não apresentem camada impermeável, pedregosa ou endurecida dentro de $25 \mathrm{~cm}$ de profundidade, uma vez que a resistência do solo à penetração afeta o crescimento das raízes das plantas. De fato, o crescimento das raízes diminui quando a resistência é maior do que $1 \mathrm{MPa}$ e reduz drasticamente quando a resistência é próxima a $5 \mathrm{MPa}$ (BENGOUGH; MULLINS, 1990; MATERECHERA et al., 1991).

Garcia (2000) verificou que 60\% do sistema radicular da bananeira "Prata-Anã" se concentra nos primeiros $30 \mathrm{~cm}$ de profundidade. Doorenbos e Kassam (1994) afirmam que o sistema radicular da bananeira não ultrapassa a profundidade de $80 \mathrm{~cm}$, sendo essa considerada suficiente, uma vez que dela se extrai a maior parte da água e que, deste total, $60 \%$ é absorvido nos primeiros $30 \mathrm{~cm}$. Em estudo de densidade do sistema radicular da bananeira cultivar Pacovan, Lacerda Filho et al. (2004) verificaram que $72,4 \%$ da densidade de comprimento das raízes se encontram nos primeiros $30 \mathrm{~cm}$ e apenas $4,6 \%$ entre 45 e $60 \mathrm{~cm}$.

Nesse sentido, o conhecimento da profundidade do solo e dos hábitos de enraizamento é fundamental para o sucesso ou não da bananicultura em determinadas áreas (ARAYA; BLANCO, 2001). Por essa razão, o presente estudo visou a verificar como os atributos radiculares da bananeira são afetados pela profundidade e pelos atributos físicos dos solos na Chapada do Apodi (CE).

\section{MATERIAL E MÉTODOS}

O estudo foi conduzido em um bananal de 24 meses, localizado na Chapada do Apodi, município de Limoeiro do Norte - CE, nas coordenadas $5^{\circ} 20^{\prime}$ de latitude sul e $38^{\circ} 5^{\prime}$ de longitude oeste. O clima da região, segundo a classificação de Köppen, é do tipo BSw'h', caracterizado por ser muito quente e semiárido. A média pluviométrica é de aproximadamente $750 \mathrm{~mm}$ por ano, e a vegetação natural é a caatinga hiperxerófila (BRASIL, 1973).

A área de estudo foi caracterizada por possuir diferenças na profundidade do solo associadas ao microrrelevo (OLIVEIRA, 2009), ocorrendo solos mais rasos na porção convexa e retilínea e solos mais profundos, na parte côncava do terreno. Para as avaliações, foram considerados dois tratamentos:um Cambissolo Háplico carbonático típico, denominado solo raso, com profundidade efetiva de 57 cm (OLIVEIRA, 2009), e um Cambissolo Háplico Ta eutrófico típico, denominado solo profundo, com profundidade efetiva de $116 \mathrm{~cm}$ (OLIVEIRA, 2009), ambos dispostos em áreas adjacentes dentro do bananal.

O bananal foi implantado em 2008 e conduzido sob as mesmas práticas de manejo nas duas áreas avaliadas neste estudo. A cultura foi estabelecida em linhas duplas, com espaçamento de 3,8 x 1,2 x 2,0 $\mathrm{m}$, totalizando 2.000 plantas por hectare. A variedade estudada foi a Willians, do subgrupo Cavendish. A irrigação foi por microaspersão, e a adubação, por meio de fertirrigação.

Para as determinações dos atributos físicos do solo e dos atributos radiculares, foram considerados dois tratamentos (solo raso e solo profundo) e seis profundidades de coleta de amostras: $0-10 ; 10-20$; $20-30 ; 30-40 ; 40-50$ e 50-60 cm. As amostras foram coletadas a uma distância de aproximadamente 25 $\mathrm{cm}$ do pseudocaule das plantas, com cinco repetições (considerando cada planta uma repetição), totalizando 60 amostras.

Amostras (60) com estrutura indeformada 
também foram coletadas com amostrador tipo Uhland (anéis de $100 \mathrm{~cm}^{3}$ ), as quais foram saturadas, pesadas, colocadas em mesa de tensão adaptada, seguindo-se a metodologia de Lima e Silva (2008), e submetidas às tensões de $10 ; 40 ; 50 ; 60$ e 100 centímetros de coluna de água (cca). Após tensão de $100 \mathrm{cca}$, as amostras foram colocadas em câmaras de pressão (KLUTE, 1986) e submetidas às tensões de 330; $700 ; 1.000$ e 1.5000 cca. Após atingir o equilíbrio para cada tensão, as amostras foram pesadas para a determinação de sua massa úmida. O modelo de van Genuchten (1980) foi utilizado para ajustar as curvas de retenção.

Ainda com as amostras indeformadas, foram determinados os valores de densidade e porosidade do solo (KLUTE, 1986). Amostras de terra fina, seca ao ar (TFSA), foram utilizadas para análise granulométrica e densidade de partículas (KLUTE, 1986).

A resistência à penetração (RP) foi determinada no campo, utilizando-se de penetrômetro de impacto, modelo comercial IAA/PLANALSUCAR/ Stolf (STOLF et al., 1983), por meio do qual foram registrados os dados de RP a cada $10 \mathrm{~cm}$, até a profundidade de $60 \mathrm{~cm}$. Ao lado da perfuração deixada pelo penetrômetro, amostras de solo foram coletadas para a determinação da umidade gravimétrica.

Para o estudo de raízes, a amostragem foi feita, utilizando-se de sonda com diâmetro interno de $4,5 \mathrm{~cm}$ e $100 \mathrm{~cm}$ de comprimento. Cada amostra coletada apresentou volume de $159 \mathrm{~cm}^{3}$. Após coleta, as amostras foram separadas e lavadas com água corrente, promovendo-se a separação entre raízes e solo. Em laboratório, as raízes lavadas foram colocadas em placas de vidro e escaneadas para obtenção de imagens. O software Safira (JORGE; OLIVEIRA RODRIGUES, 2008) foi utilizado para a análise das imagens de modo a fornecer valores referentes ao volume total, comprimento total e área superficial total de raízes por classes de diâmetro. Também foram determinadas as classes de diâmetro de raízes em cada amostra analisada.

Os dados foram submetidos ao teste de Shapiro Wilk, a 5\% de probabilidade, para a verificação da normalidade dos resíduos, sendo que houve necessidade de transformação dos dados referentes ao sistema radicular em $\mathrm{x}^{0,5}$. A análise de variância foi realizada conforme a de um delineamento inteiramente casualizado (DIC) e com parcelas subdivididas, sendo que as profundidades do solo (solo raso e profundo) foram o fator principal; e as profundidades de amostragem $(0-10 ; 10-20 ; 20-30 ; 30-40 ; 40-50$ e $50-60 \mathrm{~cm})$ foram as subdivisões. As variáveis que apresentaram diferença de médias constatadas pelo teste $\mathrm{F}$ foram submetidas ao teste de Tukey, a 5\% de probabilidade, para a comparação das mesmas.

\section{RESULTADOS E DISCUSSÃO}

No solo raso (Cambissolo Háplico carbonático típico), não houve grande variação na distribuição vertical das frações areia, silte e argila, conferindo classe textural do tipo franco-argilo-arenosa (SANTOS et al., 2005) em todas as profundidades estudadas (Tabelas 1 e 2). Apesar de não haver significância estatística, o teor de argila no solo profundo (Cambissolo Háplico Ta eutrófico típico) foi maior, conferindo uma classe textural do tipo argila (SANTOS et al., 2005).

De acordo com Oliveira (2009), o posicionamento do solo profundo na superfície côncava do terreno contribui para a maior quantidade de argila nesta área, pois superfícies côncavas no terreno geralmente condicionam fluxos convergentes de água, provocando variações na profundidade efetiva dos solos. Esses fluxos proporcionam maior taxa de intemperismo do material de origem (MONIZ; MEDINA, 1972), resultando em maiores teores de argila e profundidade. Além disso, o movimento preferencial de argila por meio de escoamento lateral da água, acumulando material proveniente da superfície convexa na superfície côncava, pode ser outra causa do aumento de argila no solo profundo. Ruth e Lennartz (2008) constataram que os conteúdos de argila e de silte foram maiores nas posições mais baixas do terreno, indicando efeito da gravidade e de processos erosivos.

Diferença significativa na densidade do solo foi encontrada entre os tipos de solo, nas camadas de 0-10 e 10-20 cm, sendo que o maior valor $\left(1,41 \mathrm{~g} \mathrm{~cm}^{-3}\right)$ foi registrado no solo raso (Tabela 2). Os valores de densidade encontrados nos dois solos estão próximos daqueles estabelecidos como ideais (REICHERT et al., 2003), exceto os valores encontrados no solo raso, nas camadas de $0-10$ e $10-20 \mathrm{~cm}$, podendo ser indicativos de início de compactação ou dos efeitos da menor profundidade efetiva do solo.

$\mathrm{O}$ maior valor de densidade de partículas (Tabela 2) foi obtido no solo raso $\left(2,82 \mathrm{~g} \mathrm{~cm}^{-3}\right)$. Em todas as profundidades dos solos, as médias da densidade de partículas estiveram acima da do quartzo $\left(2,65 \mathrm{~g} \mathrm{~cm}^{-3}\right)$, indicando a existência de partículas mais densas que elevam o valor médio encontrado.

Em relação aos maiores valores de densidade do solo, vale destacar que a compactação aumenta com a redução da macroporosidade. Além disso, há consenso na literatura de que macroporosidade inferior a $10 \%$ causa deficiência de aeração, prejudicando o sistema radicular (STOLF et al., 2011). Aplicando- 
-se esse critério aos resultados do presente estudo (Tabela 2), é possível verificar que as restrições se encontram nas camadas superficiais de ambos os solos $(0-10 \mathrm{~cm})$, pela proximidade dos valores em relação ao limite de $10 \%$. No solo profundo (mais argiloso), após a camada superficial, verifica-se decréscimo da macroporosidade, com a profundidade reduzindo-se o de $20 \%(10-20 \mathrm{~cm})$ para $12 \%(50-60$ $\mathrm{cm})$. Isso indica que a diminuição da macroporosidade, no intervalo considerado, pode estar ligada mais à gênese do solo do que à compactação provocada pelo manejo, pois os efeitos antrópicos são mais pronunciados na superfície.

Solos argilosos, mesmo compactados, podem apresentar maior porosidade total devido à maior microporosidade proporcionada pelo maior teor de argila. Comparando os resultados médios entre o solo raso (mais arenoso) e o profundo (mais argiloso), verifica-se, respectivamente: $21 \%$ e $15 \%$ (macroporosidade); $33 \%$ e $42 \%$ (microporosidade); $54 \%$ e $57 \%$ (porosidade total), ou seja, apesar de o solo mais argiloso apresentar menor macroporosidade, a porosidade total é maior devido à microporosidade.

Diferenças significativas entre solo raso e profundo foram observadas na porosidade total do solo (Tabela 2), nas camadas de 0-10; 10-20 e 20$30 \mathrm{~cm}$, sendo a maior porosidade total registrada no solo profundo $(60 \%)$. Esse solo apresentou ainda maior volume de microporos, enquanto o solo raso apresentou mais macroporos a partir dos $40 \mathrm{~cm}$ de profundidade.

Além da discussão anterior sobre os efeitos da textura na porosidade, a presença de nódulos e concreções no solo raso, registrada na descrição morfológica do perfil (OLIVEIRA, 2009), indica que a menor porosidade na camada de 10 a $20 \mathrm{~cm}$ do solo raso pode ser devida à presença deste material de granulometria mais grosseria. Segundo Cox e McFarlane (1995), a presença de cascalhos no perfil do solo pode reduzir a porosidade e a capacidade de armazenamento de água.

A retenção de água diferiu entre os solos, em todas as profundidades avaliadas (Tabela 3). Tal fato reforça a relação entre o conteúdo de água retida e a textura do solo (Tabela 2), de modo que, à medida que a textura se torna mais argilosa, aumenta também a quantidade de água retida. Além da textura, os valores referentes à porosidade também explicam o observado em relação à retenção de água. Verificou-se que, no solo raso (mais areia, mais macroporos, menos microporos e menor porosidade total), o conteúdo de água, em qualquer potencial mátrico, foi inferior ao do solo profundo (menos areia, mais microporos e maior porosidade total), sugerindo a necessidade de manejo da irrigação diferenciado entre os solos, ou seja, maior frequência de irrigação para o solo raso.

A resistência à penetração (RP), medida em campo (Figura 1a), indica adensamento nas camadas de $0-5 ; 5-10$ e $10-15 \mathrm{~cm}$ do solo raso $(1,35 ; 1,84$ e 1,77 $\mathrm{MPa}$, respectivamente), em comparação às mesmas camadas do solo profundo $(0,96 ; 1,44$ e 1,48 $\mathrm{MPa}$ ). A partir dos $30 \mathrm{~cm}$ de profundidade, a resistência do solo à penetração aumentou ainda mais no solo raso, ocorrendo RP máxima ( $5,1 \mathrm{MPa})$ aos $50 \mathrm{~cm}$ de profundidade. Como foi observada pequena variação na umidade do solo raso (Figura 1b), é provável que esse pico de resistência tenha ocorrido em função da maior frequência de nódulos e concreções (OLIVEIRA, 2009), associados, inclusive, à maior densidade de partículas a partir dos $40 \mathrm{~cm}$ de profundidade no solo raso (Tabela 2).

Estudos consideram que valores de RP superiores a $1 \mathrm{MPa}$ reduzem o crescimento de raízes que são prejudicadas mediante valores próximos a $5 \mathrm{MPa}$ (BENGOUGH; MULLINS, 1990; MATERECHERA et al., 1991). Nesse caso, pode-se considerar que os valores de RP obtidos no solo raso o tornam mais limitante ao desenvolvimento das raízes das plantas.

Com a análise dos atributos radiculares (Tabelas 4 e 5), foi constatada a presença de raízes até à profundidade $60 \mathrm{~cm}$ em ambos os solos. Mais de $70 \%$ das raízes concentraram- se nos primeiros 30 $\mathrm{cm}$ de profundidade, o que está de acordo com o mencionado por Borges e Souza (2004). No solo raso, a diminuição nos valores de área e do comprimento total de raízes foi mais acentuada a partir da camada de 30-40 cm. Já no solo profundo, essa redução foi mais acentuada a partir da camada de $40-50 \mathrm{~cm}$.

$\mathrm{O}$ solo profundo apresentou maior número de classes de diâmetro, com destaque para os primeiros $30 \mathrm{~cm}$ de profundidade (Tabela 5). Nesse solo, também foram encontradas as raízes de maior diâmetro máximo (2,2 a 6,6 mm). Nesse caso, o diâmetro máximo encontrado pode afetar diretamente o comportamento da planta, especialmente sua sustentação e longevidade. Raízes mais grossas são eficientes e importantes na sustentação da planta, minimizando prejuízos que podem ser causados pelos ventos (BORGES; SOUZA, 2004). As raízes de maior diâmetro têm menor taxa de senescência. Em parte, isso se deve à proximidade das raízes mais grossas com a parte aérea e à maior reserva de carboidratos (VOGT;BLOOMFIELD, 1991). Gill e Jackson (2000) fizeram uma revisão bibliográfica, englobando uma série de artigos sobre regeneração e longevidade de raízes em vários tipos de plantas e observaram significativo crescimento na longevidade com o aumento do diâmetro das raízes. 
No que se refere ao diâmetro mínimo, menores valores $(0,68$ a $0,79 \mathrm{~mm})$ foram encontrados no solo profundo (Tabela 4), com diferença significativa nas profundidades de 0-10 e 10-20 cm (Tabela 5). $\mathrm{O}$ diâmetro mínimo afeta diretamente a absorção de água e de nutrientes pela planta. De acordo com Rylter (1997), as raízes finas das plantas constituem um dos principais meios para acessar os recursos do solo, sendo que seu comprimento e número são indicadores da capacidade de absorção de nutrientes. Segundo o mesmo autor, as raízes finas são mais abundantes nas camadas superficiais, onde se concentram de 40 a $70 \%$ do total de biomassa dessas raízes, das quais $50-80 \%$ são compostas por biomassa morta, resultado da rápida decomposição e regeneração de raízes finas.

Supõe-se que a presença de raízes com maior variabilidade de classes de diâmetro favorece o desenvolvimento das bananeiras no solo com maior profundidade efetiva. Essa suposição é fortalecida pelos resultados de Costa et al. (2011), que avaliaram, na mesma área de estudo, a altura das plantas e o peso de cachos no segundo ciclo de produção das plantas. Os autores observaram maior altura, maior diâmetro do pseudocaule e maior peso de cachos no solo de maior profundidade efetiva.
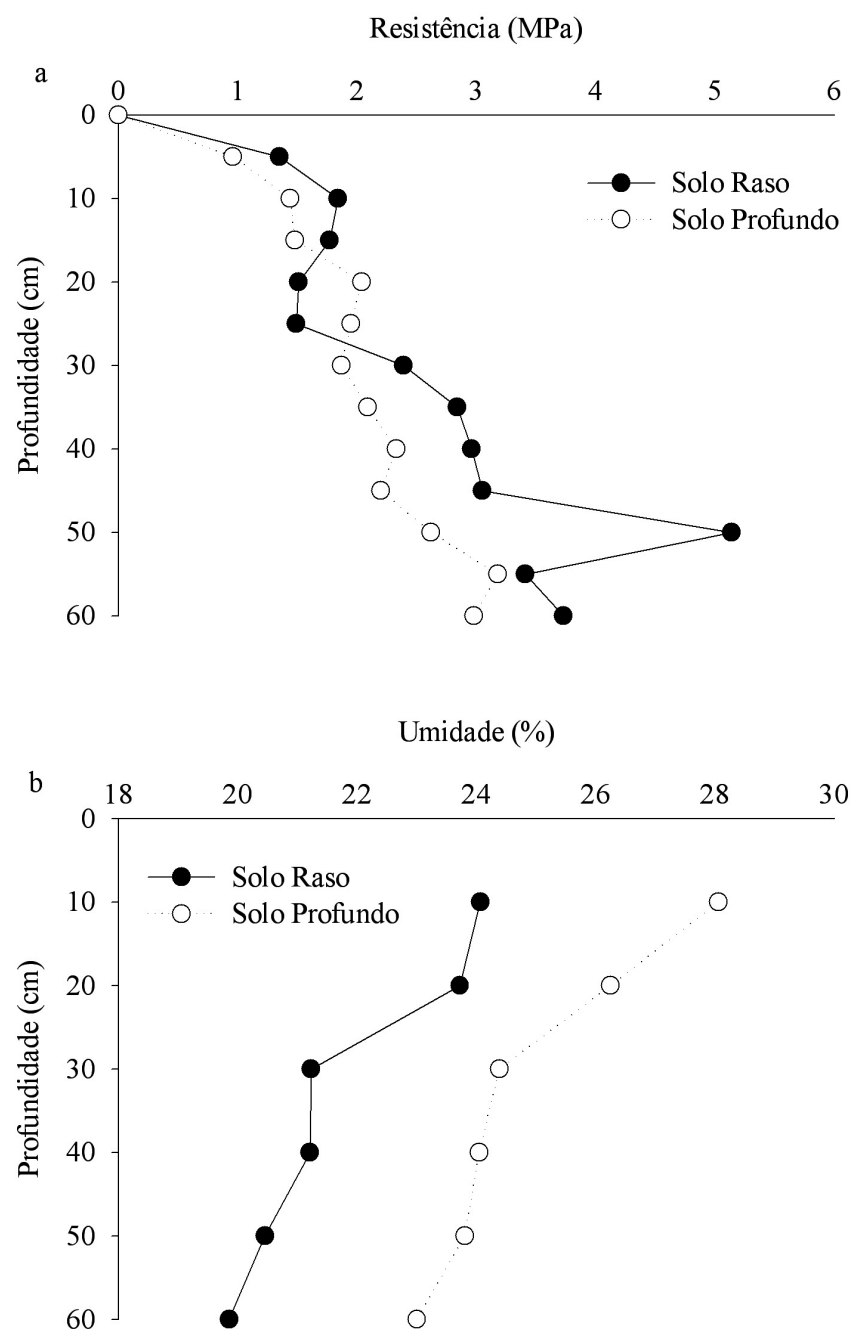

FIGURA 1 - Valores médios de resistência à penetração (a) e de umidade (b) em diferentes profundidades nos solos raso e profundo. 
TABELA 1 - Resumo do quadro da análise de variância dos atributos físicos do solo.

\begin{tabular}{|c|c|c|c|c|c|c|c|c|c|}
\hline \multirow[t]{2}{*}{$\mathrm{FV}^{(1)}$} & \multirow[t]{2}{*}{$\mathrm{GL}^{(2)}$} & \multicolumn{3}{|c|}{ Granulometria } & \multicolumn{2}{|c|}{ Densidade } & \multicolumn{3}{|c|}{ Porosidade } \\
\hline & & Areia & Silte & Argila & Solo & Partículas & Macro & Micro & Total \\
\hline & & \multicolumn{8}{|c|}{ 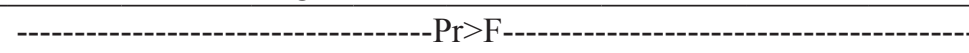 } \\
\hline Fator $\mathrm{A}^{(3)}$ & 1 & $<0,012^{*}$ & $<0,08^{\mathrm{ns}}$ & $0,37^{\mathrm{ns}}$ & $0,02^{*}$ & $0,08^{\mathrm{ns}}$ & $0,07^{\text {ns }}$ & $0,01^{* *}$ & $0,03^{*}$ \\
\hline Erro 1 & 1 & - & - & - & - & - & - & - & - \\
\hline Fator $\mathrm{B}^{(4)}$ & 5 & $0,012^{*}$ & $0,96^{\mathrm{ns}}$ & $0,13^{\mathrm{ns}}$ & $0,02^{*}$ & $0,48^{\mathrm{ns}}$ & $0,0008^{* *}$ & $0,02^{*}$ & $0,01^{*}$ \\
\hline$A \times B$ & 5 & $0,016^{*}$ & $0,99^{\text {ns }}$ & $0,50^{\text {ns }}$ & $0,05^{*}$ & $0,08^{\mathrm{ns}}$ & $0,003^{* *}$ & $0,06^{\mathrm{ns}}$ & $0,02^{*}$ \\
\hline Erro 2 & 10 & - & - & & - & - & - & - & - \\
\hline CV(\%) & & 7,7 & 14,5 & 19,6 & 7,9 & 1,5 & 28,6 & 8,6 & 6,3 \\
\hline
\end{tabular}

${ }^{(1)}$ Fonte de variação; ${ }^{(2)}$ Graus de liberdade; ${ }^{(3)}$ Profundidade efetiva do solo; ${ }^{(4)}$ Camadas de solo avaliadas; "significativo a 5\% de probabilidade; ${ }^{* *}$ significativo a $1 \%$ de probabilidade; ${ }^{\text {ns }}$ não significativo.

TABELA 2 - Valores médios de granulometria, densidade e porosidade dos solos estudados.

\begin{tabular}{|c|c|c|c|c|c|c|c|c|}
\hline \multirow[b]{2}{*}{ Prof. } & \multicolumn{3}{|c|}{ Granulometria } & \multicolumn{2}{|c|}{ Densidade } & \multicolumn{3}{|c|}{ Porosidade } \\
\hline & Areia & Silte & Argila & Solo & Partículas & Macro & Micro & Total \\
\hline $\mathrm{cm}$ & \multicolumn{3}{|c|}{ - } & \multicolumn{2}{|c|}{---- $\mathrm{g} \mathrm{cm}^{-3}-----$} & \multicolumn{3}{|c|}{---- } \\
\hline \multicolumn{9}{|c|}{ Solo raso (mais arenoso) } \\
\hline $0-10$ & $468 \mathrm{Aa}$ & 277 & 254 & $1,41 \mathrm{Aa}$ & 2,75 & $12 \mathrm{Ab}$ & $37 \mathrm{Ba}$ & $49 \mathrm{Bb}$ \\
\hline $10-20$ & $414 \mathrm{Aa}$ & 319 & 267 & $1,37 \mathrm{Aa}$ & 2,78 & $16 \mathrm{Abc}$ & $35 \mathrm{Aab}$ & $51 \mathrm{Bb}$ \\
\hline $20-30$ & $424 \mathrm{Aa}$ & 311 & 265 & 1,29 Aab & 2,78 & $21 \mathrm{Aab}$ & $32 \mathrm{Bb}$ & $54 \mathrm{Aab}$ \\
\hline $30-40$ & $410 \mathrm{Aa}$ & 340 & 251 & $1,21 \mathrm{Ab}$ & 2,76 & $24 \mathrm{Aab}$ & $32 \mathrm{Bb}$ & $56 \mathrm{Aa}$ \\
\hline $40-50$ & $428 \mathrm{Aa}$ & 316 & 256 & $1,21 \mathrm{Ab}$ & 2,79 & $27 \mathrm{Aa}$ & $29 \mathrm{Bb}$ & $57 \mathrm{Aa}$ \\
\hline $50-60$ & $421 \mathrm{Aa}$ & 331 & 248 & $1,22 \mathrm{Ab}$ & 2,82 & $27 \mathrm{Aa}$ & $30 \mathrm{Bb}$ & $57 \mathrm{Aa}$ \\
\hline Média & 428 & 315 & 257 & 1,29 & 2,78 & 21 & 33 & 54 \\
\hline \multicolumn{9}{|c|}{ Solo profundo (mais argiloso) } \\
\hline $0-10$ & $367 \mathrm{Ba}$ & 238 & 394 & $1,28 \mathrm{Ba}$ & 2,76 & $10 \mathrm{Ab}$ & $44 \mathrm{Aa}$ & $54 \mathrm{Ab}$ \\
\hline $10-20$ & $351 \mathrm{Ba}$ & 234 & 415 & $1,11 \mathrm{Bb}$ & 2,78 & $20 \mathrm{Aa}$ & $40 \mathrm{Ab}$ & $60 \mathrm{Aa}$ \\
\hline $20-30$ & $375 \mathrm{Aa}$ & 232 & 393 & 1,17 Aab & 2,72 & $17 \mathrm{Aab}$ & $40 \mathrm{Ab}$ & $57 \mathrm{Aab}$ \\
\hline $30-40$ & $268 \mathrm{Bb}$ & 338 & 394 & 1,17 Aab & 2,73 & $17 \mathrm{Aab}$ & $41 \mathrm{Aab}$ & $57 \mathrm{Aab}$ \\
\hline $40-50$ & $253 \mathrm{Bb}$ & 350 & 397 & $1,21 \mathrm{Aab}$ & 2,73 & $15 \mathrm{Bab}$ & $41 \mathrm{Aab}$ & $56 \mathrm{Aab}$ \\
\hline $50-60$ & $240 \mathrm{Bb}$ & 386 & 374 & $1,21 \mathrm{Aab}$ & 2,73 & $12 \mathrm{Bab}$ & $44 \mathrm{Aa}$ & $56 \mathrm{Aab}$ \\
\hline Média & 309 & 296 & 395 & 1,19 & 2,74 & 15 & 42 & 57 \\
\hline
\end{tabular}

Letras maiúsculas comparam médias entre solo raso e solo profundo para cada profundidade estudada. Letras minúsculas comparam médias entre profundidades nas colunas e para cada tipo de solo. Letras iguais não diferem entre si, pelo teste de Tukey, a $5 \%$ de probabilidade. 
TABELA 3 - Umidade $\left(\mathrm{m}^{3} \mathrm{~m}^{-3}\right)$ em diferentes profundidades do solo raso e profundo em função do potencial mátrico.

\begin{tabular}{|c|c|c|c|c|c|c|c|c|c|}
\hline Prof & $0 \mathrm{cca}^{(1)}$ & $10 \mathrm{cca}$ & $40 \mathrm{cca}$ & $50 \mathrm{cca}$ & $60 \mathrm{cca}$ & $100 \mathrm{cca}$ & $330 \mathrm{cca}$ & $700 \mathrm{cca}$ & $1000 \mathrm{cca} 1$ \\
\hline \multicolumn{10}{|l|}{$--\mathrm{cm}---$} \\
\hline & \multicolumn{9}{|c|}{ Umidade - Solo raso (mais arenoso) } \\
\hline 0 a 10 & $0,490 \mathrm{Ba}$ & $0,438 \mathrm{Ba}$ & $0,374 \mathrm{Ba}$ & $0,370 \mathrm{Ba}$ & $0,366 \mathrm{Ba}$ & $0,330 \mathrm{Ba}$ & $0,302 \mathrm{Ba}$ & $0,288 \mathrm{Ba}$ & $0,274 \mathrm{Ba} \quad 0,252 \mathrm{Ba}$ \\
\hline 0 a 20 & $0,510 \mathrm{Ba}$ & $0,448 \mathrm{Ba}$ & $0,360 \mathrm{Ba}$ & $0,356 \mathrm{Ba}$ & $0,346 \mathrm{Bab}$ & $0,306 \mathrm{Bab}$ & $0,270 \mathrm{Bab}$ & $0,256 \mathrm{Bab}$ & $0,242 \mathrm{Ba} 0,224 \mathrm{Bab}$ \\
\hline 20 a 30 & $0,510 \mathrm{Ba}$ & $0,438 \mathrm{Ba}$ & $0,342 \mathrm{Ba}$ & $0,334 \mathrm{Ba}$ & $0,324 \mathrm{Bab}$ & $0,29 \mathrm{Bab}$ & $0,250 \mathrm{Bab}$ & $0,238 \mathrm{Bab}$ & $0,224 \mathrm{Ba} 0,206 \mathrm{Bab}$ \\
\hline a 40 & $0,530 \mathrm{Ba}$ & $0,448 \mathrm{Ba}$ & $0,338 \mathrm{Ba}$ & $0,328 \mathrm{Ba}$ & 0,32 & 0,2 & $0,240 \mathrm{Bab}$ & $0,230 \mathrm{Bab}$ & $0,220 \mathrm{Ba} 0,1$ \\
\hline 40 a 50 & $0,510 \mathrm{Ba}$ & $0,414 \mathrm{Ba}$ & $0,314 \mathrm{Ba}$ & $0,302 \mathrm{Ba}$ & $0,292 \mathrm{Bb}$ & $0,260 \mathrm{Bb}$ & $0,226 \mathrm{Bb}$ & $0,218 \mathrm{Bab}$ & $0,202 \mathrm{Ba} \quad 0,18 \mathrm{Bb}$ \\
\hline a 60 & 0,51 & $0,412 \mathrm{Ba}$ & $0,316 \mathrm{Ba}$ & $0,306 \mathrm{Ba}$ & 0,30 & 0,2 & 0,23 & $2 \mathrm{Bb}$ & $0,204 \mathrm{Ba} 0,1$ \\
\hline \multicolumn{10}{|c|}{ Umidade - Solo profundo (mais argiloso) } \\
\hline 0 a 10 & $0,544 \mathrm{Ab}$ & $0,492 \mathrm{Aa}$ & $0,452 \mathrm{Aa}$ & 0,442Aa & $0,442 \mathrm{Aa}$ & $0,422 \mathrm{Aa}$ & $0,384 \mathrm{Aa}$ & $0,362 \mathrm{Aa}$ & $0,340 \mathrm{Aa} \quad 0,304 \mathrm{Aa}$ \\
\hline 0 a 20 & $0,604 \mathrm{Aa}$ & $0,522 \mathrm{Aa}$ & $0,424 \mathrm{Aa}$ & 0,414Aa & $0,404 \mathrm{Aa}$ & $0,374 \mathrm{Aa}$ & $0,336 \mathrm{Aa}$ & $0,314 \mathrm{Aa}$ & $0,300 \mathrm{Aa} \quad 0,270 \mathrm{Aa}$ \\
\hline 0 a 30 & 0,580Aab & $0,510 \mathrm{Aa}$ & $0,424 \mathrm{Aa}$ & 0,410Aa & $0,402 \mathrm{Aa}$ & $0,374 \mathrm{Aa}$ & $0,334 \mathrm{Aa}$ & $0,314 \mathrm{Aa}$ & $0,298 \mathrm{Aa} \quad 0,270 \mathrm{Aa}$ \\
\hline 30 a 40 & $0,546 \mathrm{Aab}$ & $0,510 \mathrm{Aa}$ & $0,428 \mathrm{Aa}$ & 0,414Aa & $0,408 \mathrm{Aa}$ & $0,372 \mathrm{Aa}$ & $0,344 \mathrm{Aa}$ & $0,32 \mathrm{Aa}$ & $0,308 \mathrm{Aa} \quad 0,274 \mathrm{Aa}$ \\
\hline 0 a 50 & $0,544 \mathrm{Ab}$ & $0,510 \mathrm{Aa}$ & $0,430 \mathrm{Aa}$ & $0,418 \mathrm{Aa}$ & $0,410 \mathrm{Aa}$ & $0,384 \mathrm{Aa}$ & $0,346 \mathrm{Aa}$ & $0,318 \mathrm{Aa}$ & $0,302 \mathrm{Aa} \quad 0,270 \mathrm{Aa}$ \\
\hline 50 a 60 & $0,562 \mathrm{Aab}$ & $0,532 \mathrm{Aa}$ & $0,458 \mathrm{Aa}$ & $0,450 \mathrm{Aa}$ & $0,442 \mathrm{Aa}$ & $0,420 \mathrm{Aa}$ & $0,384 \mathrm{Aa}$ & $0,356 \mathrm{Aa}$ & $0,336 \mathrm{Aa} \quad 0,274 \mathrm{Aa}$ \\
\hline $\mathrm{CV} \%$ & 4,89 & 6,07 & 8,13 & 8,32 & 8,41 & 10,14 & 11,31 & 12,17 & 14,43 \\
\hline
\end{tabular}

(1)Potencial mátrico 0 cca: solo saturado; cca: centímetros de coluna de água. Letras maiúsculas comparam médias entre solo raso e solo profundo para cada profundidade estudada. Letras minúsculas comparam médias entre profundidades nas colunas e para cada tipo de solo. Letras iguais não diferem entre si, pelo teste de Tukey, a 5\% de probabilidade.

TABELA 4 - Resumo do quadro da análise de variância dos atributos radiculares.

\begin{tabular}{|c|c|c|c|c|c|}
\hline $\mathrm{FV}^{(1)}$ & $\mathrm{GL}^{(2)}$ Área total Comprimento total & $\begin{array}{c}\text { Volume } \\
\text { total }\end{array}$ & $\begin{array}{l}\text { Número de } \\
\text { classes de } \\
\text { diâmetro }\end{array}$ & $\begin{array}{l}\text { Diâmetro } \\
\text { máximo }\end{array}$ & $\begin{array}{c}\text { Diâmetro } \\
\text { mínimo }\end{array}$ \\
\hline
\end{tabular}
$-\mathrm{Pr}>\mathrm{F}-$

\begin{tabular}{cccccccc} 
Fator $\mathrm{A}^{(3)}$ & 1 & $0,23^{\mathrm{ns}}$ & $0,68^{\mathrm{ns}}$ & $0,17^{\mathrm{ns}}$ & $0,053^{\mathrm{ns}}$ & $0,0002^{* *}$ & $0,1057^{\mathrm{ns}}$ \\
Erro 1 & 1 & - & - & - & - & - & - \\
${\text { Fator } \mathrm{B}^{(4)}}^{4}$ & 5 & $0,0001^{* *}$ & $0,0003^{* *}$ & $0,02^{*}$ & $0,008^{* *}$ & $<0,0001^{* *}$ & $0,0068^{* *}$ \\
$\mathrm{~A} \times \mathrm{B}$ & 5 & $0,47^{\mathrm{ns}}$ & $0,65^{\mathrm{ns}}$ & $0,69^{\mathrm{ns}}$ & $0,62^{\mathrm{ns}}$ & $0,0003^{* *}$ & $0,11^{\mathrm{ns}}$ \\
Erro 2 & 10 & - & - & - & - & - & - \\
\hline $\mathrm{CV}(\%)$ & & $34,7^{\mathrm{T}}$ & $33,7^{\mathrm{T}}$ & $53,0^{\mathrm{T}}$ & $24,1^{\mathrm{T}}$ & 31,4 & 9,5 \\
\hline
\end{tabular}

${ }^{(1)}$ Fonte de variação; ${ }^{(2)}$ Graus de liberdade; ${ }^{(3)}$ Profundidade efetiva do solo; ${ }^{(4)}$ Camadas de solo avaliadas; "significativo a $5 \%$ de probabilidade; ${ }^{* *}$ significativo a $1 \%$ de probabilidade; ${ }^{\text {ns }}$ não significativo; ${ }^{\mathrm{T}}$ transformação de dados $\left(\mathrm{x}^{0,5}\right)$ 
TABELA 5 - Atributos radiculares em seis profundidades dos solos raso e profundo.

\begin{tabular}{|c|c|c|c|}
\hline Atributos radiculares & Profundidade & Solo raso & Solo profundo \\
\hline & & \multicolumn{2}{|c|}{ Médias } \\
\hline \multirow{6}{*}{ Área total $\left(\mathrm{mm}^{2}\right)$} & 0 a 10 & $4.605,6 \mathrm{Aa}$ & $5.106,9 \mathrm{Aa}$ \\
\hline & 10 a 20 & $3.574,1 \mathrm{Aab}$ & 3.165,3 Aab \\
\hline & 20 a 30 & 3.017,4 Aabc & $1.912,6 \mathrm{Aab}$ \\
\hline & 30 a 40 & $1.814,2 \mathrm{Abc}$ & $1.924,6 \mathrm{Aab}$ \\
\hline & 40 a 50 & $1.452,6 \mathrm{Ac}$ & $1.241,1 \mathrm{Ab}$ \\
\hline & 50 a 60 & $1.327,3 \mathrm{Ac}$ & $615,7 \mathrm{Ab}$ \\
\hline \multirow{6}{*}{ Comprimento total (mm) } & 0 a 10 & $1.282,4 \mathrm{Aa}$ & $1.423,4 \mathrm{Aa}$ \\
\hline & 10 a 20 & $872,6 \mathrm{Aab}$ & 792,1 Aab \\
\hline & 20 a 30 & 797,0 Aab & $527,5 \mathrm{Aab}$ \\
\hline & 30 a 40 & $511,0 \mathrm{Ab}$ & $574,6 \mathrm{Aab}$ \\
\hline & 40 a 50 & $440,4 \mathrm{Ab}$ & $394,1 \mathrm{Ab}$ \\
\hline & 50 a 60 & $367,4 \mathrm{Ab}$ & $220,7 \mathrm{Ab}$ \\
\hline \multirow{6}{*}{ Volume total $\left(\mathrm{mm}^{3}\right)$} & 0 a 10 & $1.852,3 \mathrm{Aa}$ & $2256,0 \mathrm{Aa}$ \\
\hline & 10 a 20 & $2.020,9 \mathrm{Aa}$ & $1826,6 \mathrm{Aa}$ \\
\hline & 20 a 30 & $2.023,1 \mathrm{Aa}$ & $1083,2 \mathrm{Aa}$ \\
\hline & 30 a 40 & $891,6 \mathrm{Aa}$ & $724,4 \mathrm{Aa}$ \\
\hline & 40 a 50 & $821,8 \mathrm{Aa}$ & $401,4 \mathrm{Aa}$ \\
\hline & 50 a 60 & $593,6 \mathrm{Aa}$ & $175,6 \mathrm{Aa}$ \\
\hline \multirow{6}{*}{$\begin{array}{l}\text { Número de classes } \\
\text { (unid) }\end{array}$} & 0 a 10 & $4,2 \mathrm{Bab}$ & $9,6 \mathrm{Aab}$ \\
\hline & 10 a 20 & 6,4 Aab & $9,8 \mathrm{Aab}$ \\
\hline & 20 a 30 & $8,2 \mathrm{Aa}$ & $11,0 \mathrm{Aa}$ \\
\hline & 30 a 40 & 4,8 Aab & 6,4 Aab \\
\hline & 40 a 50 & 4,6 Aab & $6,2 \mathrm{Ab}$ \\
\hline & 50 a 60 & $3,2 \mathrm{Ab}$ & $6,0 \mathrm{Ab}$ \\
\hline \multirow{6}{*}{ Diâmetro máximo (mm) } & 0 a 10 & $2,9 \mathrm{Bb}$ & 5,3 Aab \\
\hline & 10 a 20 & $5,7 \mathrm{Ba}$ & $6,1 \mathrm{Aa}$ \\
\hline & 20 a 30 & 4,7 Bab & 6,6 Aa \\
\hline & 30 a 40 & $2,4 \mathrm{Ab}$ & $2,2 \mathrm{Ac}$ \\
\hline & 40 a 50 & $2,5 \mathrm{Ab}$ & $2,5 \mathrm{Ac}$ \\
\hline & 50 a 60 & $2,2 \mathrm{Bb}$ & $3,6 \mathrm{Abc}$ \\
\hline \multirow{6}{*}{ Diâmetro mínimo (mm) } & 0 a 10 & $0,96 \mathrm{Aa}$ & $0,79 \mathrm{Ba}$ \\
\hline & 10 a 20 & $0,78 \mathrm{Aab}$ & $0,73 \mathrm{Bab}$ \\
\hline & 20 a 30 & $0,68 \mathrm{Ab}$ & $0,68 \mathrm{Ab}$ \\
\hline & 30 a 40 & $0,73 \mathrm{Ab}$ & $0,70 \mathrm{Ab}$ \\
\hline & 40 a 50 & $0,71 \mathrm{Ab}$ & $0,70 \mathrm{Ab}$ \\
\hline & 50 a 60 & $0,68 \mathrm{Ab}$ & $0,68 \mathrm{Ab}$ \\
\hline
\end{tabular}

Médias seguidas por letras iguais, maiúsculas nas linhas e minúsculas nas colunas, não diferem entre si, pelo teste de Tukey, a 5\% de probabilidade. Obs: Valores obtidos em amostra de $159 \mathrm{~cm}^{3}$ para todos os atributos. 


\section{CONCLUSÕES}

1- Os atributos físicos do solo variam de acordo com a profundidade avaliada. O solo profundo apresenta maiores conteúdos de argila, maior volume de microporos e porosidade total, resultando em maior retenção de água quando comparado ao solo raso. Isto sugere um diferenciado manejo da irrigação entre os solos, pois o solo raso demanda maior frequência de irrigação.

2- Os demais atributos físicos (densidade do solo, densidade de partículas e resistência à penetração) são mais favoráveis ao enraizamento no solo profundo. Assim, o número de classes de diâmetro e o diâmetro máximo de raízes são maiores no solo profundo, indicando melhores condições de sustentação das plantas e maior longevidade do sistema radicular. No mesmo solo, também são encontradas raízes de menor diâmetro, representando melhores condições para a absorção de água e nutrientes, bem como melhores condições para a regeneração das bananeiras.

\section{AGRADECIMENTOS}

Ao Banco do Nordeste (BNB) pelo apoio financeiro. Ao CNPq pela concessão de bolsa mestrado ao primeiro autor. À Agrícola Famosa pela concessão da área experimental. Ao Departamento de Ciências do Solo da Universidade Federal do Ceará pela infraestrutura para realização deste trabalho.

\section{REFERÊNCIAS}

ARAYA, M.; BLANCO, F. Changes in the stratification and spatial distribution of the banana (Musa AAA cv. Grand Naine) root system of poor, regular, and good developed plants. Journal of Plant Nutrition, New York,v. 24, n. 11, p. 16791693, 2001.

BENGOUGH, A. G.; MULLINS, C. E. Mechanical impedance to root growth: a review of experimental techniques and root growth responses. The Journal of Soil Science, Oxford, v. 41, p. 341-358, 1990.

BORGES, A. L; SOUZA, L. da SILVA. O cultivo da bananeira. Cruz das Almas: Embrapa, 2004. 279 p.
BRASIL. Ministério da Agricultura. Divisão de Pesquisa Pedológica, DNPEA. Levantamento exploratório reconhecimento de solos do estado do Ceará. Recife, MA/DNPEA-SUDENE/DRN, MA/ CONTAP/ USAID/ETA, 1973. 2v, 502p. (Boletim Técnico, 28)

COSTA, M. C. G.; ALMEIDA, E. L.; FERREIRA, T. O.; OLIVEIRA, D. P.; ROMERO, R. E. Profundidade do solo e micro-relevo em bananais irrigados: impactos na nutrição mineral e potencial produtivo. Revista Ciência Agronômica, Fortaleza, v. 42, n. 3, p. 567-578. 2011.

COX, J.W.; McFARLANE, D. J. The causes of waterlogging in shallow soils and their drainage in southwestern Austrália. Journal of Hydrology, Amsterdam, v. 167, p.175-194, 1995.

DNOCS - Departamento Nacional de Obras Contra as Secas. Perímetro irrigado Jaguaribe-Apodi. Disponível em: <http://www.dnocs.gov.br/ dnocs/ doc/canais/perimetros_irrigados/ce/jaguaribe_apodi.

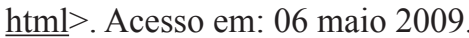

DOORENBOS, J.; KASSAM, A.H. Efeito da água no rendimento das culturas. Roma : FAO, 1994. 306p. (Irrigação e Drenagem, 33).

GARCIA, R. V. Sistema radicular de bananeira irrigada por aspersão convencional e microaspersão no Projeto Jaíba-MG. 2000. 47 f. Dissertação (Mestrado em Solos e Nutrição de Plantas) - Universidade Federal de Viçosa, Viçosa, 2000.

GILL, R. A.; JACKSON, R. B. Global patterns of root turnover for terrestrial ecosystems. New Phytologist, Cambridge, v.147, p.13-31, 2000.

JORGE, L. A. C.; OLIVEIRA RODRIGUES, A. F. de. Safira: sistema de análise de fibras e raízes. São Carlos: Embrapa Instrumentação Agropecuária, 2008. 20p. (Boletim de Pesquisa e Desenvolvimento, 24)

KLUTE, A (Ed.). Methods of soil analysis: physical and mineralogical methods. 2. ed. Madison: American Society of Agronomy, 1986. 1188 p. 
LACERDA FILHO, R.; SILVA, A.V.C.; MENDONÇA, V.; TAVARES, J.C.. Densidade do sistema radicular da bananeira 'Pacovan' sob irrigação por aspersão. Revista Brasileira de Fruticultura, Jaboticabal, v. 26, n. 3, p. 538-539, 2004.

LEMOS, M. S. S.; CURI, N.; MARQUES, J.J.G. de S. e M.; ERNESTO SOBRINHO, F. Evaluation of characteristics of Cambisols derived from limestone in low tablelands in northeastern Brazil: implications for management. Revista Brasileira de Ciência do Solo, Viçosa, MG, v. 32, n. 8, p. 825-834, 1997.

LIMA, H.V.; SILVA, A.P. Mesa de tensão com areia: procedimentos para montagem e validação. Revista Brasileira de Ciência do Solo, Viçosa, MG, v. 32, p. 2209-2214, 2008.

MATERECHERA, S. A.; DEXTER, A. R.; ALSTON, A. M. Penetration of very strong soils by seedling roots of different plant species. Plant and Soil, The Hague, v. 135, p. 31-34. 1991.

MONIZ, A. C.; MEDINA, H. P. Elementos de pedologia. São Paulo: Polígono: Editora da Universidade de São Paulo, 1972. 459 p.

OLIVEIRA, D.P. Caracaterização, gênese e mineralogia de solos da Chapada do Apodi-CE: o papel do microrrelevo. 2009. 79 f. Monografia (Graduação) - Universidade Federal do Ceará, Fortaleza, 2009.

REICHERT, J.M.; REINERT, D.J.; BRAIDA, J.A. Qualidade dos solos e sustentabilidade de sistemas agrícolas. Revista Brasileira de Ciências Ambientais, São Paulo, v. 27, p. 29-48, 2003.
RUTH, B.; LENNARTZ, B. Spatial variability of soil properties and rice yield along two catenas in Southeast China. Pedosphere, Amsterdam, v. 18, n. 4, p. 409-420, 2008.

RYLTER, R. M. Fine root production and carbon and nitrogen allocation in basket willows. 1997. Thesis (Doctoral) - Swedish University of Agricultural Sciences, Uppsala, 1997.

SANTOS, R. D.; LEMOS, R. C.; SANTOS, H. G.; KER, J. C.; ANJOS, L. H. C. Manual de descrição e coleta de solo no campo. 5. ed. Viçosa, MG, SBCS, 2005. 100 p

STOLF, R.; FERNANDES, J.; FURLANI NETO,V. L. Penetrômetro de impacto, IAA/Planalsucar-Stolf. Recomendações para o seu uso. Revista STAB, Piracicaba, v.1, n.3, p.18-23, 1983.

STOLF, R.; THURLER, A. M.; BACCHI, O. O. S.; REICHARDT, K. Method to estimate soil macroporosity and microporosity based on sand content and bulk density. Revista Brasileira de Ciência do Solo, Viçosa, MG, v. 35, p. 447-459, 2011.

VAN GENUCHTEN, M.T.H. A closed-form equation for predicting the conductivity of un saturated soils. Soil Science Society American Journal, Madison, v. 44, p. 892-897, 1980.

VOGT, K. A.; BLOOMFIELD, J. Tree root turnover and senescence. In: LASSOIE., J. P., HINCKLEY. T. M. (Ed.). Plant roots: the hidden half. New York: Marcel Dekker, 1991. p.287-305. 